\section{Localisation cérébrale}

\section{des monoamine oxydases $A$ et $B$}

La monoamine oxydase (MAO) existe sous deux formes qui different par leur préférence pour certains substrats et par leurs propriétés immunologiques. Westlund et coll. (Galveston, Texas), ont réussi à identifier les structures contenant MAO A ou B dans le cerveau de singes (Macaca fascicularis) en utilisant des anticorps monoclonaux couplés à un système de révélation à la peroxydase [I]. Ils ont pu ainsi distinguer très nettement deux types de populations neuronales: MAO A est localisée dans les groupes de cellules riches en catécholamines comme le locus niger, le locus coeruleus et la région périventriculaire de l'hypothalamus; MAO B est présente dans les régions à sérotonine, notamment l'ensemble des noyaux du raphé, et dans les astrocytes; ces conclusions sont en accord avec des travaux antérieurs effectués chez le rat.

Un résultat aussi net devrait aider à clarifier la fonction de ces enzymes dans le cerveau, y compris leur rôle hypothétique dans le contrôle du niveau des neurotransmetteurs. Au contraire, nous semblons buter ici sur un double paradoxe, physiologique et pathologique. La sérotonine est désaminée beaucoup mieux par MAO A que par MAO B, or c'est avec MAO B qu'elle coexiste. Quant à MAO A, bien qu'elle soit capable de désaminer noradrénaline et dopamine, chez le rat, on tend à admettre que, chez l'homme du moins, elle serait peu active'in vivo et la dopamine serait surtout métabolisée par la forme B [2]. On est donc conduit à supposer que les MAO auraient surtout comme fonction de protéger leurs neurones respectifs contre l'intrusion d'amines exogènes, neurotransmettrices ou non.

Sur le plan pathologique, on a invoqué l'action de ces enzymes pour expliquer la neurotoxicité d'une drogue, le MPTP (contaminant d'une "héroïne de synthèse ", qui provoque l'apparition de symptô- le singe $\left(\mathrm{m} / \mathrm{s} n^{\circ} 2\right.$ vol. $i, p .104$ et [3]). Le produit toxique est en fait le MPP : il résulte de l'oxydation du MPTP par la forme B de la MAO exclusivement. Le paradoxe est ici que les neurones à sérotonine, seuls à contenir MAOB, ne sont pas affectés par le toxique, alors qu'est électivement atteinte la substance noire qui contient la dopamine mais pas de MAOB. L'interprétation serait que des structures à sérotonine, en contact étroit avec la substance noire ou le striatum (noyau dorsal du raphé), ou des astrocytes qui contiennent MAOB, oxyderaient le MPTP en MPP; celui-ci serait ensuite libéré, puis capté sélectivement par les neurones à dopamine, comme le suggèrent les travaux de Javitch et coll. [3].

Les recherches sur la localisation des MAO dans le cerveau posent donc autant de questions qu'elles en résolvent; elles ont cependant le mérite de conduire à éviter de tirer des conclusions formelles d'observations insuffisamment étayées.

J.-C. D.

1. Westlund KM, Denney RM, Kochersperger LM, Abell CW. Distinct monoamine oxidase A and $B$ populations in primate brain. Science 1985 ; 230: $18 \mathrm{I}-3$.

2. Glover V, Sandler M, Owen F, Riley J. Dopamine is a monoaminc oxidasc $B$ substrate in man.

3. Javitch JA, D'Amato R, Strittmatter SM, Snyder SH. Parkinsonism-inducing ncurotoxin, $\mathrm{N}$-methyl-4-phenyl-i;2,3,6-tetrahydropyridinc: uptake of the metabolite $\mathrm{N}$-methyl 4-phenyl pyridine by dopamine neurons explains selective toxicity. Proc Natl Acad Sci USA 1985; 82: 2173-7.

* Différenciation terminale $=$ Stade ultime d'un processus de différenciation aboutissant à une cellule incapable de se diviser.

I. Noda M, Ko M, Ogura A, et al. Sarcoma virus carrying ras oncogenes induce differenciationassociated propertics in a neuronal cell line Nature $1985 ; 318: 73-5$.

2. Alema S, Casalborc $P$, Agostini E, Tato F. Differentiation of $\mathrm{PC}_{12}$ phacochromocytoma cells induced by $v$-src oncogenc. Nature 1985; 316: 557-9.

3. Brugge JS, Cotton P. C, Qucral AE, et al. Neurones express high levels of a structurally modificd activated form of $\mathrm{pp}^{60^{c}-\mathrm{stre}}$. Nature 1985; 316: 554-7. Nature 1977, 265: 80-I.

\section{Quand les oncogènes se comportent comme des différenciateurs}

Beaucoup reste à faire pour comprendre les mécanismes d'action des oncogènes sur le métabolisme et la prolifération cellulaire. On savait déjà que de nombreux oncogènes avaient une claire spécificité tissulaire d'action, transformant tel type de cellules et non tel autre. Dans le cas des cellules nerveuses, c'est à un effet plus paradoxal encore que l'on assiste: certains oncogènes, non seulement ne sont pas transformants, mais vont jusqu'à bloquer la synthèse d'ADN et induisent la différenciation " terminale "* de la cellule. Ceci a été observé pour la lignée PC 12 dérivée de phéochromocytome de rat. Ces cellules se divisent indéfiniment en culture et répondent au facteur de croissance NGF (nerve gromth factor) par une cessation de la division cellulaire et l'expression de propriétés caractéristiques des cellules sympathiques différenciées. L'infection par des virus porteurs des oncogènes v-Ki-ras, v-Ha-ras, v-src produit le même effet $[I, 2]$. L'oncogène c-src et son produit, la phosphoprotéine $p p^{60^{\circ-s r c}}$, semblent d'ailleurs jouer un rôle important dans la différenciation cérébrale au cours de laquelle ils sont intensément_exprimés [3]. La protéine $p p^{60^{c-s n c}}$ ainsi détectée dans les neurones mitotiques semble par ailleurs légèrement différente de celle synthétisée à un très faible taux dans d'autres cellules [3].

Ainsi, tel gène transformant pour certaines cellules est-il probablement impliqué dans la différenciation normale d'autres types cellulaires, ayant même la propriété de bloquer la division cellulaire. Ces résultats indiquent ainsi que, contrairement $\grave{a}$ une idée répandue, la fonction des produits d'oncogènes peut n'être pas limitée à la stimulation de la prolifé- ration cellulaire.

A. $\mathbf{K}$. 